\title{
Kompetenzen im Alltag richtig interpretieren
}

\section{Angesichts steigender Heilmittelverordnungen untersuchten deutsche Kinderärzte, wie es um die Alltagskompetenz von Vorschulkindern bestellt ist, und ob ein Zusammenhang zwischen erfolgreichem Schulstart und dem Beherrschen dieser Alltagsfertigkeiten besteht.}

A llen Eltern, die im 3. Quartal 2005 die große kinder- und jugendärztlichen Praxis des Autors in Wilhelmshaven aufsuchten, wurde ein Fragebogen zur Alltagskompetenz ihrer zwischen 1998 und 2001 geborenen Kinder (Patienten und gesunde Geschwisterkinder) sowie ein Fragebogen mit soziodemografischen Angaben überreicht. Fragebögen zu 373 Kindern im Alter von 4-7 Jahren konnten ausgewertet werden. Im ersten Fragebogen wurden 35 Alltagskompetenzen erfragt, die nach Ansicht der Autoren relevant waren, wie z. B. sich vollständig an- und ausziehen, Schleife binden, Tisch decken, sich ein Brot selbst schmieren oder sich am Telefon mit Namen melden.

Parallel dazu wurden Eltern von 250 Schulanfängern am ersten Elternabend gebeten, identische Fragebögen auszufüllen. Sechs Wochen später wurden die Lehrer gebeten, neun Bereiche bei diesen Kindern zu beurteilen: Umsetzung von mündlichen Aufforderungen, Arbeitsverhalten, mündliche Mitarbeit, Graphomotorik, räumliche Orientierung, Lebenspraxis, Sozialverhalten, emotionale Stabilität und Auffassungsgabe.

Die Studienpopulation bestand $\mathrm{zu}$ $51,1 \%$ aus Mädchen und zu 48,9\% aus Jungen. 98,4\% hatten die deutsche Staatsbürgerschaft. 10,5\% der Praxiskinder und 12,8\% der Schulkinder hatten Ergotherapie erhalten.

Die Befragung ergab, dass im Alter von 4,5-5,5 Jahren nur gut ein Drittel sein eigenes Geschirr und Besteck nach dem Essen abräumt. Zwei Drittel der Kinder können sich selbstständig anziehen und mit Messer und Gabel essen. Laut Denver-Test müssten sich $90 \%$ selbstständig anziehen können.

Im Alter von sieben Jahren konnten sich weitgehend alle Kinder selbstständig an- und ausziehen, 90\% ihr Brot selbst schmieren und $88 \%$ eine Schleife binden. Hilfe im Haushalt (Wäsche aufhängen

Alltägliche Handlungen von Kindern, wie das Anziehen, sollten gefördert werden. und falten, abtrocknen) wurde nur bei der Hälfte der Kinder angegeben. Die Werte der Praxiskinder unter den 7-Jährigen entsprachen denen der Schulanfänger.

Mit Hilfe einer multiplen Regressionsanalyse konnte ermittelt werden, dass die väterliche Bildung und die Alltagsfertigkeiten schwach signifikant mit einer positiven Lehrereinschätzung korrelierten. Vorschulische Förderangebote, worunter wohl auch Ergotherapie fällt, hatten hingegen keinen positiven Einfluss auf die Lehrerbeurteilung. Unter den Alltagsfertigkeiten war es vor allem das Ausmaß der Mithilfe im Haushalt, das positiv mit dem Lehrerurteil korrelierte. Bei Kenntnis der väterlichen Schulbildung und des Scores aus den Alltagskompetenzen konnte bei 66,3\% der Kinder eine korrekte Vorhersage für einen gelungenen Schulstart erfolgen.

Die Autoren folgern aus ihren Ergebnissen, dass viele Kinder alltagspraktische Erfahrungen versäumen. Sie stellen die Hypothese auf, dass Alltagskompetenzen in gleicher Weise wie die elterliche Schulbildung für einen gelungenen Schulstart bedeutsam sind.

Kommentar: Die Kinder haben im Lauf der Jahrzehnte an Alltagskompetenzen verloren - jedenfalls bis zum Einschulalter. Dies ist in bestimmten Bereichen

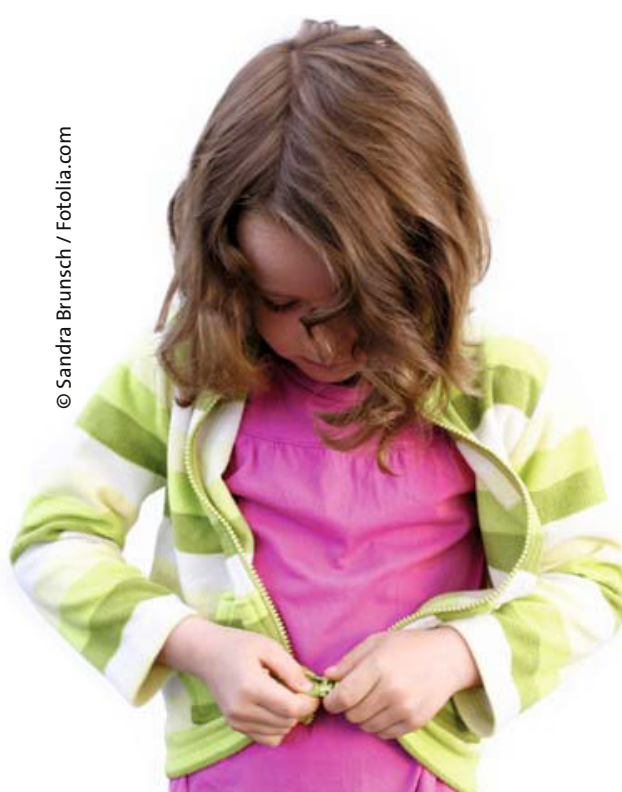

auch schwer zu verhindern, da beispielsweise die meisten Kinderschuhe mit Klettverschlüssen versehen sind. Die Studie bestätigt Tatsachen aus anderen Untersuchungen über den Erwerb von motorischen Fertigkeiten: Ein vielfaches Wiederholen ist erforderlich, um einen Handlungsablauf zu erlernen. Nur tägliches Verrichten von bestimmten Aktivitäten, wie z. B sich selbst anziehen oder im Haushalt helfen, führt dazu, diese Alltagsfertigkeiten auch zu beherrschen.

Die Autoren selbst räumen ein, dass aufwendigere Studien erforderlich seien, um den Zusammenhang zwischen erfolgreichem Schulstart und dem Erlangen von Alltagskompetenzen zu erfassen. Die fehlenden sensomotorischen Erfahrungen könnten zu einem erschwerten Schulstart führen. Es könnten aber auch die Ursachen für den misslungenen Schulstart bereits im Vorschulalter zu einer schwächeren Alltagskompetenz führen. Neben einer direkten Kausalität wäre auch denkbar, dass Entwicklungsdefizite dazu führen, dass die Kinder sowohl im Bereich der Alltagskompetenzen Mühe haben als auch der Schulstart erschwert ist, ohne dass es einen kausalen Zusammenhang zwischen den beiden Komponenten gibt.

Auch ein therapeutischer Nutzen kann durch die vorliegende Studie nicht geklärt werden. Vorschulische Förderangebote waren nicht effektiv - aber waren es die richtigen? Geht man von fehlenden sensomotorischen Erfahrungen als Ursache aus, so wäre vor allem von einer Beratung der Eltern - am besten schon begleitend ab Geburt - am meisten zu erhoffen, z. B. durch den Kinder- und Jugendarzt oder durch Elternkurse.

Auch wenn die Studie viele Fragen offen lässt, so kann die Schlussfolgerung uneingeschränkt unterstützt werden: Die Möglichkeiten der häuslichen Förderung sollten mehr genutzt werden. Die Partizipation von Kindern an alltäglichen Verrichtung sollte empfohlen werden. Ob damit die Kinder weniger Schulprobleme haben werden - dies zu klären, bleibt weiteren Studien überlassen.

Dr. Kisten Stollhoff

Dernick R et al. Kindliche Kompetenzen im Alltag (KIKA) und gelungener Schulstart. Kinderärztliche Praxis 2009; 80:350-7 